Vol. 10 (2): 385-388 (2020)

\title{
SENSITIVITY OF CASE DEFINITIONS IN SYNDROMIC SURVEILLANCE
}

\author{
Artan Simaku*, Eugena Tomini, Elona Kureta, Adela Vasili, Eduard Kakarriqi, Silva Bino \\ *Institute of Public Health, Tirana, Albania; \\ *Corresponding Author Artan Simaku, email: artan.simaku@gmail.com;
}

Received May 2019; Accepted September 2019; Published May 2020;

DOI: https://doi.org/10.31407/ijees10.219

\begin{abstract}
Syndromic surveillance is a primary health care-facility and emergency room (ER)-based syndromic surveillance system aiming at detecting outbreaks and undertaking public health actions. It is based on weekly notify cations of nine syndromes by over 1,600 General Practitioners (GPs) in the 36 districts of Albania. Data is aggregated by district epidemiologists (DE) and centralized by the national Institute of Public Health. A syndrome is "a set of symptoms or condones that occur together and suggest the presence of a certain disease or an increased chance of developing the disease." In the context of syndromic surveillance, a syndrome is a set of non-specific pre-diagnosis medical and other informal on that may indicate the release of a bioterrorism agent or natural disease outbreak. Since its inception, syndromic surveillance has mainly focused on early event detection: gathering and analyzing data in advance of diagnostic case confirmation to give early warning of a possible outbreak. The system is useful for detecting and responding to natural disease outbreaks, and thus they have the potential to significantly advance and modernize the practice of public health surveillance
\end{abstract}

Keywords: public health, early event detection, sensitivity 